www.nature.com/pj

\title{
Nanosized palladium-catalyzed Suzuki-Miyaura coupling polymerization: synthesis of soluble aromatic poly(ether ketone)s
}

\author{
Katsuya Maeyama ${ }^{1}$, Tadashi Tsukamoto ${ }^{1}$, Masanori Suzuki ${ }^{2}$, Shuhei Higashibayashi ${ }^{3}$ and Hidehiro Sakurai ${ }^{3}$ \\ Nanosized palladium species, which were prepared in situ from $\mathrm{Pd}(\mathrm{OAc})_{2}, \mathrm{PPh}_{3}$ and $\mathrm{Bu}_{4} \mathrm{NOAc}$ in 1,4-dioxane, were suitable \\ for the synthesis of $1,1^{\prime}$-binaphthyl-2,2'-dioxy-bearing aromatic poly(ether ketone)s 5 . The resulting poly(ether ketone)s 5 are \\ soluble in typical organic solvents, such as $\mathrm{CHCl}_{3}$, tetrahydrofuran and $\mathrm{N}, \mathrm{N}$-dimethylformamide. Glass transition temperatures $\left(T_{\mathrm{g}}\right.$ 's) \\ of poly(ether ketone)s 5 are in the range of $188-224{ }^{\circ} \mathrm{C}$. Temperatures where $10 \%$ weight losses of poly(ether ketone)s 5 occur \\ under $\mathrm{N}_{2}$ flow are in the range of $445-480^{\circ} \mathrm{C}$.
}

Polymer Journal (2013) 45, 401-405; doi:10.1038/pj.2012.158; published online 29 August 2012

Keywords: Suzuki-Miyaura coupling polymerization; nanosized palladium; aromatic poly(ether ketone)s; 1,1'-binaphthyls

\section{INTRODUCTION}

Aromatic poly(ether ketone)s, which consist of aromatic, carbonyl and ether units, have drawn considerable attention owing to their outstanding chemical and physical stability. ${ }^{1-3}$ General synthetic methods of aromatic poly(ether ketone)s are mainly (i) nucleophilic aromatic substitution polymerization of aromatic difluorides with aromatic diols, (ii) electrophilic aromatic substitution polymerization of highly electron-rich aromatics with aromatic dicarboxylic acids/ acid dichlorides and (iii) transition metal-catalyzed aromatic coupling polymerization, such as $\mathrm{NiBr}_{2} / \mathrm{Zn}$-mediated aromatic homo-coupling polymerization of aromatic dichlorides. ${ }^{2,3}$ Almost all of the reported aromatic poly(ether ketone)s have been synthesized through nucleophilic aromatic substitution polymerization owing to high conversion, easy treatment and wide application. As ether bonds are inevitably formed through this polymerization, they behave as flexible units and lower the thermal stability of the resulting polymers. On the other hand, aromatic carbonyl bonds are formed in electrophilic aromatic substitution polymerization. However, selection of polymerizable and regioselective electron-rich arenes is extremely limited. Although aromatic-aromatic bonds are formed in $\mathrm{NiBr}_{2} / \mathrm{Zn}$-mediated aromatic homo-coupling polymerization, strict treatment against oxygen and moisture during polymerization is required for smooth polymerization.

We have reported the synthesis of several aromatic polyketones using these three methods. ${ }^{4-11}$ Among these studies, it has been revealed that aromatic polyketones containing twisted aromatic ringassembly units show both excellent thermal stability and sufficient solubility to typical organic solvents, affording flexible and thermally stable polymer films. In particular, introduction of $1,1^{\prime}$-binaphthyl6,6'-diyl units to polyketone backbones was effective for the development of aromatic polyketones with both high $T_{\mathrm{g}}$ and sufficient solubility through these three methods. ${ }^{8-11}$

The Suzuki-Miyaura cross-coupling reaction is one of the most useful protocols for aromatic-aromatic bond formation reactions and has been applied for the synthesis of a lot of $\pi$-conjugated organic materials, including polymers. ${ }^{12-15}$ Although it has the advantage of high conversion and easy treatment, it has scarcely been applied to the synthesis of aromatic polyketones. In particular, Suzuki-Miyaura coupling reaction of bis(halobenzoyl)-type monomers requires long reaction times, probably because of lower reactivity based on carbonyl groups. ${ }^{15}$

Recently, we presented a preliminary report on the successful synthesis of aromatic poly(ether ketone)s through nanosized palladium-catalyzed ${ }^{16-26}$ Suzuki-Miyaura coupling polymerization of $2,2^{\prime}$-bis(iodobenzoyl)-1,1'-binaphthyls with aromatic diboric acid pinacol esters. ${ }^{27}$ In this article, we would like to discuss the nanosized palladium-catalyzed Suzuki-Miyaura coupling polymerization from the viewpoints of the scope and limitations of monomer structures and detail an investigation of reaction conditions.

\section{EXPERIMENTAL PROCEDURE}

\section{Materials}

(S)-BINOL $\left(1,[\alpha]_{\mathrm{D}}^{25}:-33^{\circ}\right)$, triphenylphosphine and trimethyl borate were purchased from Kanto Chemical Co. Ltd. (Tokyo, Japan). $\mathrm{Pd}(\mathrm{OAc})_{2}$, potassium carbonate and pinacol were purchased from Wako Pure Chemical Industries

\footnotetext{
${ }^{1}$ Department of Polymer Science and Engineering, Graduate School of Science and Engineering, Yamagata University, Yonezawa, Yamagata, Japan; ${ }^{2}$ Department of Organic and Polymer Materials Chemistry, Graduate School of Technology, Tokyo University of Agriculture and Technology, Koganei, Tokyo, Japan and ${ }^{3}$ Research Center for Molecular Scale Nanoscience, Institute for Molecular Science, Myodaiji, Okazaki, Japan

Correspondence: Professor K Maeyama, Department of Polymer Science and Engineering, Graduate School of Science and Engineering, Yamagata University, 4-3-16 Jonan, Yonezawa, Yamagata 992-8510, Japan.

E-mail: maeyama@yz.yamagata-u.ac.jp

Received 21 June 2012; revised 17 July 2012; accepted 18 July 2012; published online 29 August 2012
} 
(Osaka, Japan). $\mathrm{Bu}_{4} \mathrm{NOAc}$ and MS4A were purchased from Aldrich (St Louis, MO, USA). These reagents were used as received. 1,4-Dioxane was purchased from Kanto Chemical Co. Ltd. and was used after distillation in the presence of $\mathrm{CaH}_{2}$. Dimethylformamide was purchased from Kanto Chemical Co. Ltd. and was used after distillation in the presence of $\mathrm{CaH}_{2}$ under reduced pressure.

4-Fluoro-4'-iodobenzophenone (2A) and related halobenzophenones $2 \mathrm{~B}-2 \mathrm{E}$ were prepared through $\mathrm{AlCl}_{3}$-mediated Friedel-Crafts acylation reaction of fluorobenzene with the corresponding halobenzoyl chloride. Diboric acid pinacol esters $4 \mathrm{~m}$ and $\mathbf{4 p}$ were prepared through esterification of the corresponding diboric acids with pinacol. The diboric acids were prepared through lithiation of the corresponding dibromide followed by transmetallation by trimethyl borate and acidic workup.

\section{Instruments}

${ }^{1} \mathrm{H}-\mathrm{NMR}$ spectra were recorded on a JEOL ECX-400 (JEOL, Tokyo, Japan, $400 \mathrm{MHz})$ and a JEOL AL-300 $(300 \mathrm{MHz})$. Chemical shifts are expressed in p.p.m. relative to the internal standard of $\mathrm{Me}_{4} \mathrm{Si}(\delta=0.00) .{ }^{13} \mathrm{C}$-NMR spectra were recorded on a JEOL ECX-400 (100 MHz) and a JEOL AL-300 (75 MHz). Chemical shifts are expressed in p.p.m. relative to the internal standard of $\mathrm{CDCl}_{3}(\delta=77.0)$. Infrared (IR) measurement was recorded on a JEOL FTIR4100 and a Horiba FT-210 (Horiba, Kyoto, Japan). Molar rotations $\left([\Phi]_{25}^{\mathrm{D}}\right)$ were estimated on the basis of the measurement in a $\mathrm{CHCl}_{3}$ solution $\left(1 \mathrm{~g} \mathrm{dl}^{-1}\right)$ using a JASCO DIP-1000 digital polarimeter (JASCO, Tokyo, Japan). Circular dichroism measurement was performed in a $\mathrm{CHCl}_{3}$ solution $\left(6.6 \times 10^{-5}\right.$ moll ${ }^{-1}$ ) using a JASCO J-720WI. Gel permeation chromatography measurements were carried out at a flow rate of $1.0 \mathrm{ml} \mathrm{min}^{-1}$ at $40^{\circ} \mathrm{C}$ using $\mathrm{CHCl}_{3}$ as an eluent on a JASCO PU-2080 equipped with a Ultraviolet detector $(254 \mathrm{~nm})$ and a Shodex K-804L column (Shodex, Tokyo, Japan). Glass transition temperatures $\left(T_{\mathrm{g}}\right)$ were estimated on the basis of differential scanning calorimetry thermograms. The differential scanning calorimetry thermograms were recorded on a PerkinElmer DSC-4000 differential scanning calorimeter with a heating rate of $10 \mathrm{~K} \mathrm{~min}^{-1}$ (Waltham, MA, USA). Thermal degradation temperatures $\left(T_{\mathrm{d}}\right)$ were estimated on the basis of thermogravimetric analysis thermograms. The thermogravimetric analysis thermograms were recorded on a PerkinElmer TGA 4000 thermogravimeter with a heating rate of $10 \mathrm{~K} \mathrm{~min}^{-1}$.

\section{Synthesis of diiodide $3 \mathrm{P}(\mathrm{I}), 3 \mathrm{M}(\mathrm{I}) 3 \mathrm{O}(\mathrm{I})$, dibromide $3 \mathrm{P}(\mathrm{Br})$ and dichloride $3 \mathrm{P}(\mathrm{Cl})$}

To a 50-ml two-necked flask, (S)-BINOL (1, 1.14 g, $4.0 \mathrm{mmol})$, 4-fluoro-4' iodobenzophenone $(2 \mathrm{~A}, 4.54 \mathrm{~g}, 14 \mathrm{mmol})$, anhydrous potassium carbonate $(1.66 \mathrm{~g}, 12 \mathrm{mmol})$ and freshly distilled dimethylformamide $(5 \mathrm{ml})$ were added. The mixture was stirred at $150^{\circ} \mathrm{C}$ for $24 \mathrm{~h}$. The reaction mixture was poured into water in a beaker. The precipitate was collected by suction filtration and extracted with $\mathrm{CHCl}_{3}$ three times. The combined extracts were dried over anhydrous $\mathrm{MgSO}_{4}$. After removal of the drying reagent, $\mathrm{CHCl}_{3}$ was removed under reduced pressure. The crude product was purified by silica gel column chromatograph (hexane:chloroform $=1: 1)$ to yield diiodide $3 \mathbf{P}(\mathbf{I})$ in $71 \%$ yield $(2.55 \mathrm{~g})$ as a white powder. A series of dihalides 3 were also prepared in the same manner.

Diiodide 3P(I): Yield: 71\%. ${ }^{1} \mathrm{H}-\mathrm{NMR} \delta\left(400 \mathrm{MHz}, \mathrm{CDCl}_{3}\right): 6.79(4 \mathrm{H}, \mathrm{d}$, $J=8 \mathrm{~Hz}), 7.27-7.33(6 \mathrm{H}, \mathrm{m}), 7.36(4 \mathrm{H}, \mathrm{d}, J=8 \mathrm{~Hz}), 7.45(2 \mathrm{H}, \mathrm{t}, J=8 \mathrm{~Hz})$, $7.52(4 \mathrm{H}, \mathrm{d}, J=8 \mathrm{~Hz}), 7.78(4 \mathrm{H}, \mathrm{d}, J=8 \mathrm{~Hz}), 7.91(2 \mathrm{H}, \mathrm{d}, J=8 \mathrm{~Hz}), 7.95(2 \mathrm{H}$, d, $J=8 \mathrm{~Hz})$ p.p.m. ${ }^{13} \mathrm{C}-\mathrm{NMR} \delta\left(100 \mathrm{MHz}, \mathrm{CDCl}_{3}\right): 99.7,117.4,120.0,122.9$, $125.5,126.0,127.1,128.3,130.4,131.0,131.2,131.3,132.0,134.2,137.2,137.6$, 151.1, 161.6 and 194.2 p.p.m. IR $v(\mathrm{KBr}): 1648,1587,1500$ and $1240 \mathrm{~cm}^{-1}$. HRMS-FAB $(m / z)$ For $\mathrm{C}_{46} \mathrm{H}_{28} \mathrm{I}_{2} \mathrm{O}_{4}$ : Calcd $\left[\mathrm{M}^{+}\right]$: 898.0082. Found: 898.0096. $[\alpha]_{\mathrm{D}}^{25}:-24^{\circ} \cdot[\Phi]_{\mathrm{D}}^{25}:-216^{\circ}$.

Diiodide $3 \mathrm{M}(\mathrm{I})$ : Yield: $61 \% .{ }^{1} \mathrm{H}-\mathrm{NMR} \delta\left(400 \mathrm{MHz}, \mathrm{CDCl}_{3}\right): 6.81(4 \mathrm{H}, \mathrm{d}$, $J=8 \mathrm{~Hz}), 7.17(2 \mathrm{H}, \mathrm{t}, J=8 \mathrm{~Hz}), 7.27-7.35(6 \mathrm{H}, \mathrm{m}), 7.45(2 \mathrm{H}, \mathrm{t}, J=8 \mathrm{~Hz})$, $7.55(4 \mathrm{H}, \mathrm{d}, J=8 \mathrm{~Hz}), 7.58(2 \mathrm{H}, \mathrm{d}, J=8 \mathrm{~Hz}), 7.85(2 \mathrm{H}, \mathrm{d}, J=8 \mathrm{~Hz}), 7.91(2 \mathrm{H}, \mathrm{d}$, $J=8 \mathrm{~Hz}), 7.96(2 \mathrm{H}, \mathrm{d}, J=8 \mathrm{~Hz}), 7.97(2 \mathrm{H}$, s $)$ p.p.m. ${ }^{13} \mathrm{C}-\mathrm{NMR} \delta(100 \mathrm{MHz}$, $\left.\mathrm{CDCl}_{3}\right)$ : 94.2, 117.4, 120.1, 123.0, 125.5, 126.0, 127.1, 128.3, 129.0, 130.0, 130.4, 131.0, 131.1, 132.2, 134.1, 138.4, 140.0, 140.9, 151.1, 161.7 and 193.6 p.p.m. IR $v(\mathrm{KBr}): 1654,1587,1500$ and $1243 \mathrm{~cm}^{-1}$. HRMS-FAB $(\mathrm{m} / \mathrm{z})$ for $\mathrm{C}_{46} \mathrm{H}_{28} \mathrm{I}_{2} \mathrm{O}_{4}$ : Calcd $\left[\mathrm{M}^{+}\right]$: 898.0082 . Found: 898.0096. $[\alpha]_{\mathrm{D}}^{25}:-38^{\circ}[\Phi]_{\mathrm{D}}^{25}:-341^{\circ}$.

Diiodide 3O(I): Yield: $83 \% .{ }^{1} \mathrm{H}-\mathrm{NMR} \delta\left(400 \mathrm{MHz}, \mathrm{CDCl}_{3}\right): 6.77(4 \mathrm{H}, \mathrm{d}$, $J=8 \mathrm{~Hz}), 7.13(2 \mathrm{H}, \mathrm{t}, J=8 \mathrm{~Hz}), 7.20-7.34(6 \mathrm{H}, \mathrm{m}), 7.38-7.47(6 \mathrm{H}, \mathrm{m}), 7.54$
$(4 \mathrm{H}, \mathrm{d}, J=8 \mathrm{~Hz}), 7.85-7.96(6 \mathrm{H}, \mathrm{m})$ p.p.m. ${ }^{13} \mathrm{C}-\mathrm{NMR} \delta\left(100 \mathrm{MHz}, \mathrm{CDCl}_{3}\right)$ : $92.3,117.7,119.9,122.8,125.5,125.9,127.1,127.9,128.3,128.4,130.0,130.3$, $131.0,132.5,134.1,139.7,144.2,151.8,162.2$ and 195.9 p.p.m. IR $v(\mathrm{KBr})$ : $1664,1585,1500$ and $1243 \mathrm{~cm}^{-1} \cdot[\alpha]_{\mathrm{D}}^{25}:-32^{\circ} \cdot[\Phi]_{\mathrm{D}}^{25}:-287^{\circ}$.

Dibromide 3P(Br): Yield: 83\%. ${ }^{1} \mathrm{H}-\mathrm{NMR} \delta\left(400 \mathrm{MHz}, \mathrm{CDCl}_{3}\right): 6.68(4 \mathrm{H}, \mathrm{d}$, $J=8 \mathrm{~Hz}), 7.30(4 \mathrm{H}, \mathrm{d}, J=8 \mathrm{~Hz}), 7.32(2 \mathrm{H}, \mathrm{t}, J=8 \mathrm{~Hz}), 7.46(2 \mathrm{H}, \mathrm{t}, J=8 \mathrm{~Hz})$, 7.49-7.57 $(12 \mathrm{H}, \mathrm{m}), 7.91(2 \mathrm{H}, \mathrm{d}, J=8 \mathrm{~Hz}), 7.95(2 \mathrm{H}, \mathrm{d}, J=8 \mathrm{~Hz})$ p.p.m. ${ }^{13} \mathrm{C}-\mathrm{NMR} \delta\left(100 \mathrm{MHz}, \mathrm{CDCl}_{3}\right): 117.4,120.0,122.9,125.5,126.0,127.1,127.2$, $128.3,130.4,131.0,131.2,131.4,131.6,132.0,134.1,136.6,151.1,161.6$ and 194.2 p.p.m. IR $v(\mathrm{KBr}): 1654,1587,1500,1245$ and $1068 \mathrm{~cm}^{-1}$. HRMS-FAB $(m / z)$ for $\mathrm{C}_{46} \mathrm{H}_{28} \mathrm{Br}_{2} \mathrm{O}_{4}$ : Calcd $\left[\mathrm{M}^{+}\right]: 802.0356$. Found: 802.0362. $[\alpha]_{\mathrm{D}}^{25}:-49^{\circ}$. $[\Phi]_{\mathrm{D}}^{25}:-393^{\circ}$.

Diiodide 3P(Cl): Yield: 90\%. ${ }^{1} \mathrm{H}-\mathrm{NMR} \delta\left(300 \mathrm{MHz}, \mathrm{CDCl}_{3}\right): 6.80(4 \mathrm{H}, \mathrm{d}$, $J=8.7 \mathrm{~Hz}), 7.29-7.35(6 \mathrm{H}, \mathrm{m}), 7.39-7.48(6 \mathrm{H}, \mathrm{m}), 7.53(4 \mathrm{H}, \mathrm{d}, J=8.7 \mathrm{~Hz})$, $7.59(4 \mathrm{H}, \mathrm{d}, J=8.4 \mathrm{~Hz}), 7.92(2 \mathrm{H}, \mathrm{d}, J=8.4 \mathrm{~Hz}), 7.96(2 \mathrm{H}, \mathrm{d}, J=8.7 \mathrm{~Hz})$ p.p.m. ${ }^{13} \mathrm{C}-\mathrm{NMR} \delta\left(75 \mathrm{MHz}, \mathrm{CDCl}_{3}\right): 117.3,119.8,122.8,125.3,125.8,126.9$, $128.2,128.5,130.2,130.9,131.1,131.2,131.9,134.0,136.1,138.5,151.0,161.4$ and 194.0 p.p.m. IR $v(\mathrm{KBr}): 1656,1590,1243$ and $1090 \mathrm{~cm}^{-1}$. HRMS-FAB $(m / z)$ for $\mathrm{C}_{46} \mathrm{H}_{28} \mathrm{Cl}_{2} \mathrm{O}_{4}$ : Calcd $\left[\mathrm{M}^{+}\right]$: 714.1366. Found: 714.1379. $[\alpha]_{\mathrm{D}}^{25}$ : $-4.1^{\circ} \cdot[\Phi]^{25}:-29^{\circ}$.

\section{Synthesis of aromatic polyketones 5}

To a dried 30-ml two-necked flask equipped with a reflux condenser, palladium acetate $(4.5 \mathrm{mg}, 20 \mu \mathrm{mol})$ and triphenylphosphine $(10.5 \mathrm{mg}$, $40 \mu \mathrm{mol})$ were added. Tetrabutylammonium acetate $(603 \mathrm{mg}, 2.0 \mathrm{mmol})$, pottasium carbonate $(276 \mathrm{mg}, 2.0 \mathrm{mmol})$, molecular sieves $4 \mathrm{~A}(70 \mathrm{mg})$, diiodide $3 \mathbf{P}(\mathbf{I})(180 \mathrm{mg}, 0.2 \mathrm{mmol})$, boric acid pinacol ester $4 \mathrm{~m}(77.9 \mathrm{mg}$, $0.2 \mathrm{mmol})$ and 1,4-dioxane $(1.0 \mathrm{ml})$ were added to this flask. After stirring at $100^{\circ} \mathrm{C}$ for $1 \mathrm{~h}$, the reaction mixture was poured into $150 \mathrm{ml}$ of $\mathrm{MeOH} /$ aqueous $\operatorname{HCl}(\mathrm{v} / \mathrm{v}=9 / 1)$ in a beaker. The precipitant was washed with water and acetone. The solid was dissolved in $\mathrm{CHCl}_{3}$ and filtrated by celite. After removal of $\mathrm{CHCl}_{3}$ under reduced pressure, polyketone $5 \mathrm{Pm}$ was obtained in $90 \%$ yield $(140.4 \mathrm{mg})$. Other polyketones were synthesized in the same manner.

Polyketone 5Pm: ${ }^{1} \mathrm{H}-\mathrm{NMR} \delta\left(400 \mathrm{MHz}, \mathrm{CDCl}_{3}\right): 3.84(6 \mathrm{H}, \mathrm{s}), 6.60(1 \mathrm{H}, \mathrm{s})$, $6.83(4 \mathrm{H}, \mathrm{d}, J=8 \mathrm{~Hz}), 7.22-7.35(10 \mathrm{H}, \mathrm{m}), 7.38-7.51(3 \mathrm{H}, \mathrm{m}), 7.51-7.74(8 \mathrm{H}$, m), 7.84-8.00 (4H, m) p.p.m. ${ }^{13} \mathrm{C}-\mathrm{NMR} \delta\left(100 \mathrm{MHz}, \mathrm{CDCl}_{3}\right): 56.0,96.2$, $117.5,120.0,122.3,122.9,125.4,126.0,127.0,128.3,129.3,129.9,130.4,131.0$, $132.0,132.2,132.8,134.2,135.9,142.1,151.4,157.7,161.3$ and 195.1 p.p.m. IR $v(\mathrm{KBr}): 1654,1600,1498,1234$ and $1203 \mathrm{~cm}^{-1} \cdot[\alpha]_{\mathrm{D}}^{25}:-5.8^{\circ}[\Phi]_{\mathrm{D}}^{25}:-45^{\circ}$.

Polyketone 5Pp: Yield 91\%. ${ }^{1} \mathrm{H}-\mathrm{NMR} \delta\left(400 \mathrm{MHz}, \mathrm{CDCl}_{3}\right): 3.78(6 \mathrm{H}, \mathrm{s})$, $6.85(4 \mathrm{H}, \mathrm{d}, J=8 \mathrm{~Hz}), 6.98(2 \mathrm{H}, \mathrm{s}), 7.26-7.36(10 \mathrm{H}, \mathrm{m}), 7.40-7.48(2 \mathrm{H}, \mathrm{m})$, 7.60-7.70(4H, m), 7.73(4H, d, $J=8 \mathrm{~Hz}), 7.86-7.99(4 \mathrm{H}, \mathrm{m})$ p.p.m. ${ }^{13} \mathrm{C}-\mathrm{NMR} \delta$ $\left(100 \mathrm{MHz}, \mathrm{CDCl}_{3}\right): 56.5,114.7,117.5,120.0,122.9,125.4,126.0,127.0,128.3$, $129.4,129.8,130.0,130.3,131.0,131.9,132.2,134.2,136.5,142.2,150.9,151.4$, 161.3 and 195.0 p.p.m. IR $v(\mathrm{KBr}): 1654,1600,1498,1236,1211 \mathrm{~cm}^{-1} \cdot[\alpha]_{\mathrm{D}}^{25}$ : $-5.9^{\circ} .[\Phi]_{\mathrm{D}}^{25}:-46^{\circ}$.

Polyketone 5Mm: Yield 49\%. ${ }^{1} \mathrm{H}-\mathrm{NMR} \delta\left(400 \mathrm{MHz}, \mathrm{CDCl}_{3}\right): 3.75(6 \mathrm{H}, \mathrm{s})$, $6.57(1 \mathrm{H}, \mathrm{s}), 6.81(4 \mathrm{H}, \mathrm{d}, J=8 \mathrm{~Hz}), 7.19-7.44(6 \mathrm{H}, \mathrm{m}), 7.50-7.68(11 \mathrm{H}, \mathrm{m})$, 7.78-7.93(8H, m) p.p.m. ${ }^{13} \mathrm{C}-\mathrm{NMR} \delta\left(100 \mathrm{MHz}, \mathrm{CDCl}_{3}\right): 55.9,96.0,117.5$, $120.0,122.3,122.9,125.4,125.9,127.0,128.1,128.3,130.3,131.0,131.9,132.3$, 132.7, 133.1, 134.1, 137.7, 138.0, 151.4, 157.2, 161.3 and 195.4 p.p.m. IR $v$ $(\mathrm{KBr}): 1654,1585,1500,1232$ and $1203 \mathrm{~cm}^{-1} \cdot[\alpha]_{\mathrm{D}}^{25}:-6.6^{\circ} \cdot[\Phi]_{\mathrm{D}}^{25}:-51^{\circ}$.

Polyketone 5Mp: Yield $63 \% .{ }^{1} \mathrm{H}-\mathrm{NMR} \delta\left(400 \mathrm{MHz}, \mathrm{CDCl}_{3}\right): 3.71(6 \mathrm{H}, \mathrm{s})$, $6.84(4 \mathrm{H}, \mathrm{d}, J=8 \mathrm{~Hz}), 6.94(2 \mathrm{H}, \mathrm{s}), 7.22-7.32(4 \mathrm{H}, \mathrm{m}), 7.34-7.43(4 \mathrm{H}, \mathrm{m})$, $7.46(2 \mathrm{H}, \mathrm{t}, J=8 \mathrm{~Hz}), 7.61(2 \mathrm{H}, \mathrm{d}, J=8 \mathrm{~Hz}), 7.67(4 \mathrm{H}, \mathrm{d}, J=8 \mathrm{~Hz}), 7.72(2 \mathrm{H}, \mathrm{d}$, $J=8 \mathrm{~Hz}), 7.82-7.95(6 \mathrm{H}, \mathrm{m})$ p.p.m. ${ }^{13} \mathrm{C}-\mathrm{NMR} \delta\left(100 \mathrm{MHz}, \mathrm{CDCl}_{3}\right): 56.5$, $114.6,117.4,120.0,122.9,125.4,125.9,127.0,128.1,128.3,128.7,129.8,130.3$, $130.8,130.9,131.8,132.3,133.2,134.2,137.8,138.3,150.7,151.3,161.4$ and 195.3 p.p.m. IR $\vee(\mathrm{KBr}): 1654,1587,1500,1234$ and $1211 \mathrm{~cm}^{-1}$. [ $\left.\alpha\right]_{\mathrm{D}}^{25}:-4.5^{\circ}$. $[\Phi]_{\mathrm{D}}^{25}:-35^{\circ}$.

\section{RESULTS AND DISCUSSION}

\section{Preparation of monomers 3}

According to our previous papers, ${ }^{4,9}$ three regioisomeric diiodides $3 \mathbf{M}(\mathbf{I})$, $3 \mathrm{P}(\mathrm{I})$ and $\mathbf{3 O}(\mathrm{I})$ were prepared via nucleophilic aromatic substitution reaction of $(S)$-BINOL (1) with 4-fluoroiodobenzophenones 2A-C, 
which are prepared through Friedel-Crafts acylation of fluorobenzene with iodobenzoyl chloride in the presence of $\mathrm{AlCl}_{3}$, in moderate yields (Scheme 1). The corresponding dibromide $\mathbf{2 D}$ and dichloride $2 \mathrm{E}$ were also prepared in the same procedure.

\section{Optimization of reaction conditions-polymerization between monomer $3 \mathrm{P}(\mathrm{I})$ and $4 \mathrm{~m}$}

Polymerization of monomer $3 \mathbf{P}(\mathbf{I})$ with diboric acid pinacol ester $4 \mathrm{~m}$ was conducted under the various reaction conditions. The results are shown in Table 1 . When the nanosized palladium catalyst, ${ }^{16}$ which was prepared in situ from $\mathrm{Pd}(\mathrm{OAc})_{2}, \mathrm{PPh}_{3}$ and $\mathrm{Bu}_{4} \mathrm{NOAc}$ in 1,4-dioxane, was employed, smooth and rapid polymerization proceeded to give poly(ether ketone) 5Pm. As shown in runs 1-3, $10 \mathrm{~mol} \% \mathrm{Pd}(\mathrm{OAc})_{2}(=20 \mu \mathrm{mol})$ against the monomers $(0.2 \mathrm{mmol}$, respectively) were the most suitable to obtain high-molecular-weight polymers. When $20 \mathrm{~mol} \%$ catalyst was employed, the molecular weights of the resulting polymer slightly decreased, probably owing to aggregation of nanosized palladium under concentrated conditions (run 3). Even during polymerization in the presence of $10 \mathrm{~mol} \%$ catalyst, it seems that active nanosized palladium species are gradually deactivated by slow aggregation. Consequently, prolongation of reaction time gave no effects on increase in molecular weights. When $\mathrm{Bu}_{4} \mathrm{NOAc}$, was not added, that is, under general palladium catalyst system (conditions B), only low-molecular-weight polymer was obtained even after stirring for $6 \mathrm{~h}$ (run 4). Use of a more polar solvent, that is, dimethylformamide was also ineffective under the general palladium catalyst system (run 5). On the other hand, addition of $\mathrm{Bu}_{4} \mathrm{NBr}$ was also effective to enable smooth polymerization (run 6). When $\mathrm{Pd}\left(\mathrm{PPh}_{3}\right)_{4}$ was employed instead of $\mathrm{Pd}(\mathrm{OAc})_{2} /$ $2 \mathrm{PPh}_{3}$, the moderate-molecular-weight polymer was obtained.
Consequently, the $\mathrm{Pd}(\mathrm{OAc})_{2} / \mathrm{PPh}_{3} / \mathrm{Bu}_{4} \mathrm{NOAc}$ system (run 2) was determined as the optimized reaction conditions.

Synthesis of aromatic poly(ether ketone)s 5

At first, polymerization of the corresponding dibromide $3 \mathbf{P}(\mathbf{B r})$ and dichloride $3 \mathrm{P}(\mathrm{Cl})$ with diboric acid pinacol ester $4 \mathrm{~m}$ was carried out under the above optimized conditions. Although the corresponding poly(ether ketone) $\mathbf{5} \mathbf{P m}$ was obtained, the molecular weights of the polymers derived from $3 \mathbf{P}(\mathbf{B r})$ and $3 \mathbf{P}(\mathbf{C l})$ were lower than those derived from $3 \mathbf{P}(\mathbf{I})$. In the case of dibromide $3 \mathbf{P}(\mathbf{B r})$ and dichloride $3 \mathbf{P}(\mathbf{C l})$, the oxidation addition step proceeds slower as imagined, affording lower-molecular-weight polymer. However, it could be evaluated that the nanosized palladium-catalyzed polymerization of dichloride $3 \mathbf{P}(\mathbf{C l})$ proceeded moderately (Table 2 ).

Next, polymerization of $m$-substituted and $o$-substituted diiodides, $3 \mathbf{M}(\mathbf{I})$ and $\mathbf{3 O}(\mathrm{I})$, were also performed. When $m$-substituted diiodide $3 \mathbf{M}(\mathrm{I})$ was used, poly(ether ketone)s $5 \mathrm{Mm}$ and $5 \mathrm{Mp}$ with lower molecular weights were obtained. When $o$-substituted diiodide 30 (I) was used, no polymerization proceeded and only diiodide $\mathbf{3 O}$ (I) was recovered. These reactions demonstrate that steric hindrance around reactive halogens influences the reactivity in the transmetallation step and the $o$-substitution structure is more disadvantageous.

All of the poly(ether ketone)s $5 \mathrm{Pm}, 5 \mathrm{Pp}, 5 \mathrm{Mm}$ and $5 \mathrm{Mp}$ were soluble in typical solvents as shown in Table 3. Among these polymers, poly(ether ketone)s $5 \mathrm{Mm}$ and $5 \mathrm{Mp}$, which are derived from $m$-substiutited diiodide $3 \mathbf{M}(\mathbf{I})$, were more soluble than $5 \mathrm{Pm}$ and $5 \mathrm{Pp}$. The difference of the solubility is probably because of not only the decrease of molecular weights but also the bent structures based on $m$-substitution compared with linear ones based on p-substitution.

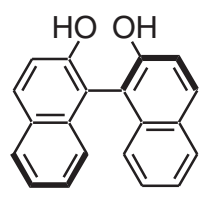

$(S)-1$

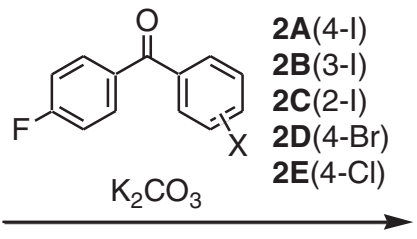

DMF, $150^{\circ} \mathrm{C}, 24 \mathrm{~h}$
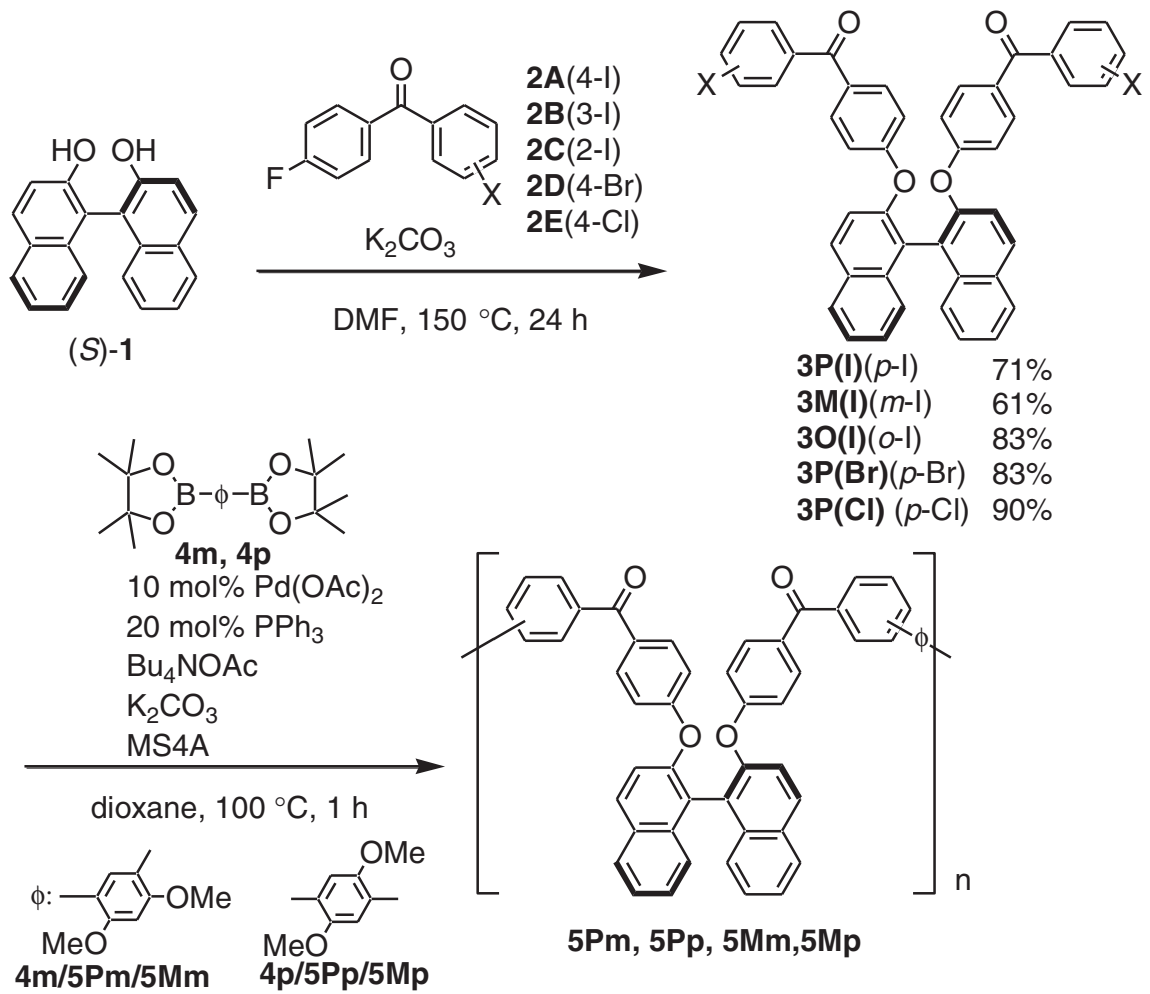

Scheme 1 Suzuki-Miyaura coupling polymerization of dihalides 3 with diboric acid pinacol esters $4 \mathrm{~m}$ and $4 \mathrm{p}$. 
Table 1 Nanosized palladium-catalyzed Suzuki-Miyaura coupling polymerization of diiodide $3 \mathrm{P}(\mathrm{I})$ with diboric acid pinacol ester $4 \mathrm{~m}$

\begin{tabular}{lllllrrr} 
Run Conditions & $\mathrm{Pd}(\mathrm{OAC})_{2}(\mu \mathrm{mol})$ & Time $(\mathrm{h})$ & Yield (\%) & $M n^{\mathrm{a}}$ & $M w^{\mathrm{a}}$ & $M w / M n^{\mathrm{a}}$ \\
\hline 1 & $\mathrm{~A}^{\mathrm{b}}$ & 10 & 1 & 82 & 8900 & 16500 & 1.85 \\
2 & $\mathrm{~A}^{\mathrm{b}}$ & 20 & 1 & 90 & 10100 & 16600 & 1.64 \\
3 & $\mathrm{~A}^{\mathrm{b}}$ & 40 & 1 & 90 & 7000 & 15300 & 2.19 \\
4 & $\mathrm{~B}^{\mathrm{c}}$ & 40 & 6 & 83 & 3200 & 8400 & 2.58 \\
5 & $\mathrm{C}^{\mathrm{d}}$ & 40 & 6 & 42 & 4600 & 10000 & 2.15 \\
6 & $\mathrm{D}^{\mathrm{e}}$ & 20 & 1 & 79 & 10800 & 21300 & 1.96 \\
7 & $\mathrm{E}^{\mathrm{f}}$ & 20 & 1 & 87 & 6200 & 14700 & 2.36
\end{tabular}

aEstimated by gel permeation chromatography (eluent; $\mathrm{CHCl}_{3}$ ) based on polystyrene standards. Reaction conditions A: monomer $3 \mathrm{P}(\mathrm{I})$ and $4 \mathrm{~m}(0.2 \mathrm{mmol}$, respectively), palladium acetate, $\mathrm{PPh}_{3}$ (twice against $\mathrm{Pd}(\mathrm{OAc})_{2}$ ), potassium carbonate (2 mmol), Bu $\mathrm{NOAc}_{4}(2 \mathrm{mmol}), \mathrm{MS} 4 \mathrm{~A}$ (70 $\mathrm{mg})$ and 1,4-dioxane $(1 \mathrm{ml})$, reflux, $1 \mathrm{~h}$.

'Reaction conditions B: Bu NOAC and MS4A were not added against conditions A.

dReaction conditions $\mathrm{C}$ : In replace of dioxane, dimethylformamide was used. Other reaction conditions are the same as conditions $\mathrm{B}$.

eReaction conditions $\mathrm{D}$ : In replace of $\mathrm{Bu}_{4} \mathrm{NOAc}, \mathrm{Bu}_{4} \mathrm{NBr}$ was used. Other reaction conditions are the same as conditions $A$.

fReaction conditions $\mathrm{E}$ : In replace of $\mathrm{Pd}(\mathrm{OAc})_{2}$ and $2 \mathrm{PPh}_{3}, \mathrm{Pd}\left(\mathrm{PPh}_{3}\right)_{4}$ was used. Other reaction conditions are the same as conditions $A$.

Table 2 Synthesis of aromatic poly(ether ketone)s $5^{\text {a }}$

\begin{tabular}{lccrrrr}
\hline 3 & 4 & 5 & Yield/\% & \multicolumn{1}{c}{$M_{n}{ }^{\mathrm{b}}$} & \multicolumn{1}{c}{$M_{w}{ }^{\mathrm{b}}$} & $M_{w} / M_{n}{ }^{\mathrm{b}}$ \\
\hline $3 \mathrm{P}(\mathrm{I})$ & $4 \mathrm{~m}$ & $5 \mathrm{Pm}$ & 90 & 10100 & 16600 & 1.64 \\
$3 \mathrm{P}(\mathrm{Br})$ & $4 \mathrm{~m}$ & $5 \mathrm{Pm}$ & 51 & 2100 & 7000 & 3.33 \\
$3 \mathrm{P}(\mathrm{Cl})$ & $4 \mathrm{~m}$ & $5 \mathrm{Pm}$ & 60 & 1500 & 4900 & 3.28 \\
$3 \mathrm{P}(\mathrm{I})$ & $4 \mathrm{p}$ & $5 \mathrm{Pp}$ & 91 & 5400 & 16700 & 3.09 \\
$3 \mathrm{M}(\mathrm{I})$ & $4 \mathrm{~m}$ & $5 \mathrm{Mm}$ & 49 & 2100 & 7600 & 3.59 \\
$3 \mathrm{M}(\mathrm{I})$ & $4 \mathrm{p}$ & $5 \mathrm{Mp}$ & 63 & 3200 & 10500 & 3.27 \\
$30(\mathrm{I})$ & $4 \mathrm{~m}$ & - & $0^{c}$ & - & - & - \\
\hline
\end{tabular}

aReaction conditions: monomer 3 and $\mathbf{4}(0.2 \mathrm{mmol}$, respectively), palladium acetate $(20 \mu \mathrm{mol})$, $\mathrm{PPh}_{3}(40 \mu \mathrm{mol})$, potassium carbonate $(2 \mathrm{mmol}), \mathrm{Bu}_{4} \mathrm{NOAc}(2 \mathrm{mmol}), \mathrm{MS} 4 \mathrm{~A}(70 \mathrm{mg})$ and 1,4-dioxane ( $1 \mathrm{ml})$, reflux, $1 \mathrm{~h}$.

beatimated by gel permeation chromatography (eluent; $\mathrm{CHCl}_{3}$ ) based on polystyrene standards. ${ }^{\mathrm{c}}$ No polymerization.

\section{Thermal properties}

Thermogravimetric analysis of poly (ether ketone)s $\mathbf{5}$ proved that they have excellent thermal stability. No loss of the weights in the temperature ranging up to ca. $450{ }^{\circ} \mathrm{C}$ under $\mathrm{N}_{2}$ flow was observed. These thermal behaviors are similar to the polyketones bearing methoxy groups, which indicates that the cleavage of $\mathrm{O}-\mathrm{CH}_{3}$ bonds occurs firstly during thermal degradation.

Glass transition temperatures $\left(T_{\mathrm{g}}\right)$ of poly(ether ketone)s $\mathbf{5}$ are in the range of $188-224^{\circ} \mathrm{C}$, which are higher than those of PEEK $\left(143^{\circ} \mathrm{C}\right)$ and almost all of the reported aromatic poly(ether ketone)s including our previous works. ${ }^{4-10}$ The excellent thermal stability and sufficient solubility depend on non-coplanar structures of $1,1^{\prime}$-binaphthyl-2,2'-diaryoxy units, which give suitable suppression of the free rotation and the $\pi, \pi$-stacking to polymer main chains. In addition, the exclusion of flexible ether units from the main chains is another factor to increase $T_{\mathrm{g}}$.

\section{Optical properties}

Optical rotation of poly (ether ketone)s 5 was measured in a $1-\mathrm{g} \mathrm{dl}^{-1}$ $\mathrm{CHCl}_{3}$ solution. Specific rotation $[\alpha]$ and molar rotation $[\Phi]$ of poly (ether ketone)s $\mathbf{5}$ are shown in Table 4. There is only negligible difference among these values. Figure 1 shows circular dichroism spectra of monomers $3 \mathbf{P}(\mathbf{I})$ and $\mathbf{3 M}(\mathbf{I})$ derived from $(S)-\mathbf{1}$, and
Table 3 Solubility of aromatic poly(ether ketone)s $5^{a}$

\begin{tabular}{lcccccc}
\hline 5 & $\mathrm{MeOH}$ & $\mathrm{CHCl}_{3}$ & THF & DMSO & DMF & NMP \\
\hline $5 \mathrm{Pm}$ & - & ++ & +- & +- & ++ & ++ \\
$5 \mathrm{Pp}$ & - & ++ & +- & + & ++ & ++ \\
$5 \mathrm{Mm}$ & - & ++ & ++ & ++ & ++ & ++ \\
$5 \mathrm{Mp}$ & - & ++ & ++ & ++ & ++ & ++
\end{tabular}

Abbreviations: DMF, N,N-dimethylformamide; DMSO, dimethyl sulfoxide; NMP, $\mathrm{N}$-methylpyrrolidinone; THF, tetrahydrofuran.

${ }^{a}(++)$, soluble at room temperature; $(+)$, soluble on heating; $(+-)$, partially soluble; $(-)$, insoluble.

Table 4 Thermal and optical properties of aromatic poly(ether ketone)s 5

\begin{tabular}{lcccc}
\hline 5 & $T_{g}\left({ }^{\circ} \mathrm{C}\right)^{\mathrm{a}}$ & $T_{d 10}\left({ }^{\circ} \mathrm{C}\right)^{\mathrm{b}}$ & {$[\alpha]_{D}^{25}(\text { degree })^{\mathrm{c}}$} & {$[\Phi]^{25}$ (degree $^{c}$} \\
\hline $5 \mathrm{Pm}$ & 224 & 445 & -5.8 & -45 \\
$5 \mathrm{Pp}$ & 205 & 465 & -5.9 & -46 \\
$5 \mathrm{Mm}$ & 188 & 480 & -6.6 & -51 \\
$5 \mathrm{Mp}$ & 191 & 475 & -4.5 & -35 \\
\hline
\end{tabular}

Determined on the basis of differential scanning calorimetry (DSC) curves. Heating rate: $10 \mathrm{Kmin}^{-1}$.

bTemperature where a $10 \%$ weight loss occurs. Heating rate: $10 \mathrm{~K} \mathrm{~min}^{-1}$

Measured in a $\mathrm{CHCl}_{3}$ solution ( $1 \mathrm{gdl}^{-1}$ ).

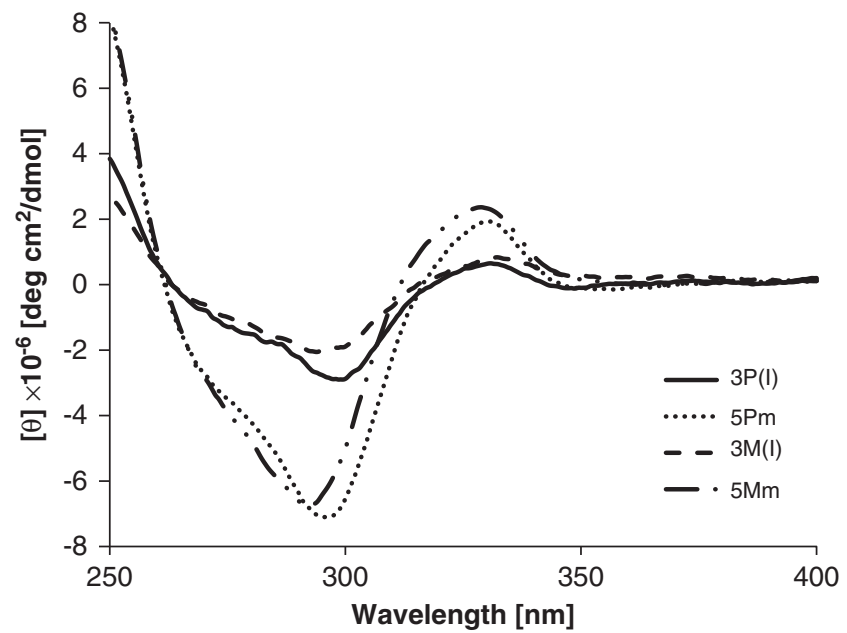

Figure 1 Circular dichroism spectra of monomers $\mathbf{3}$ and poly(ether ketone)s $\mathbf{5}$.

poly(ether ketone)s $\mathbf{5 P m}$ and $\mathbf{5} \mathbf{M m}$ derived from the $(S)$-monomers. Although larger cotton effects in the spectra of $3 \mathrm{M}(\mathrm{I})$ and $5 \mathrm{Mm}$ were observed than those of $\mathbf{3 P}(\mathbf{I})$ and $\mathbf{5 P m}$, there are no differences between the diiodides and the corresponding polymers. These results probably indicate that the poly(ether ketone)s $\mathbf{5}$ hold no specific regular secondary structures.

\section{CONCLUSIONS}

Nanosized palladium species, which are easily prepared in situ from $\mathrm{Pd}(\mathrm{OAc})_{2}, \mathrm{PPh}_{3}$ and $\mathrm{Bu}_{4} \mathrm{NOAc}$ in 1,4-dioxane, were suitable for Suzuki-Miyaura cross-coupling polymerization of diiodides 3 and aromatic diboric acid pinacol esters 4 to synthesize 1,1'-binaphthyl$2,2^{\prime}$-diaryloxy-bearing aromatic poly(ether ketone)s 5 . This catalyst was able to be applied to polymerization of aromatic dibromide and 
even aromatic dichloride. Addition of ammonium salt, that is, $\mathrm{Bu}_{4} \mathrm{NOAc}$ and $\mathrm{Bu}_{4} \mathrm{NBr}$, was requisite for smooth polymerization. The resulting poly(ether ketone)s $\mathbf{5}$ are soluble in typical organic solvents, such as $\mathrm{CHCl}_{3}$, tetrahydrofuran and dimethylformamide. Poly(ether ketone)s $\mathbf{5}$ are optically active on the basis of optically active 1,1'-binaphthyl-2,2'-oxy units. Glass transition temperatures $\left(T_{\mathrm{g}}^{\prime} \mathrm{s}\right)$ of poly(ether ketone)s 5 are in the range of $188-224^{\circ} \mathrm{C}$. Temperatures where $10 \%$ weight losses of poly(ether ketone)s 5 occur under $\mathrm{N}_{2}$ flow are in the range of $445-480^{\circ} \mathrm{C}$.

\section{ACKNOWLEDGEMENTS}

This work is supported by the Joint Studies Programs (2011-2012) of the Institute for Molecular Science.

1 Staniland, P. A., Eastmond, G. C., Ledwith, A., Russo, S. \& Sigwalt, P. (ed.) Comprehensive Polymer Science vol. 5, 483 (Pergamon Press, Oxford, UK, 1989).

2 Maeyama, K. Synthesis of wholly aromatic polyketones based on fine molecular designing. J. Synth. Org. Chem. 69, 616-626 (2005).

3 Maeyama, K. Aromatic poly(ether ketone)s functional materials. Expected Mater. Future 8, 42-47 (2008).

4 Maeyama, K., Tagata, Y. \& Yonezawa, N. 2,2'-Bis(4-benzoylphenoxy)biphenyl: a novel efficient acyl-acceptant monomer yielding wholly aromatic polyketones via friedelcrafts type polymerization with arenedicarbonyl chloride. Polym. J. 36, 146-150 (2004).

5 Maeyama, K., Tagata, Y., Nishimori, Y., Yamazaki, M., Maruyama, A. \& Yonezawa, N. Effective synthesis of wholly aromatic polyketones using 2.2'-diaryloxybiphenyl and arenedicarboxylic acid derivatives via friedel-crafts type acylation polymerization. React. Funct. Polym. 61, 71-79 (2004).

6 Maeyama, K., Ohe, T., Nakamura, H. \& Yonezawa, N. Successful synthesis of fully aromatic polyketones via nickel complex-mediated aromatic coupling polymerization of bis(chlorobenzoylated) o-terphenyls. Polym. J. 35, 290-292 (2003).

7 Maeyama, K., Ohe, T., Nakamura, H. \& Yonezawa, N. Synthesis of fully aromatic polyketones without ether linkages in the main chain. nickel complex-mediated aromatic coupling polymerization of bis(chlorobenzoylated) o-terphenyls. Polym. J. 35, 1009-1015 (2003).

8 Maeyama, K., Hikiji, I., Ogura, K., Okamoto, A., Ogino, K., Saito, H. \& Yonezawa, N. Synthesis of optically active aromatic poly(ether ketone)s via nucleophilic aromatic substitution polymerization. Polym. J. 37, 707-710 (2005).

9 Maeyama, K., Maeda, S., Ogino, K., Saito, H. \& Yonezawa, N. Synthesis of optically active aromatic poly(ether ketone)s containing 2,2'-bis(4-benzoylphenoxy)-1,1'binaphthyl-6,6'-ene backbones. React. Funct. Polym. 65, 229-237 (2005).

10 Maeyama, K., Ogura, K., Okamoto, A., Ogino, K., Saito, H. \& Yonezawa, N. Nickel complex-mediated synthesis of optically active wholly aromatic polyketones bearing 2,2'-dimethoxy-1,1'-binaphthylene units. Polym. J. 37, 736-741 (2005).
11 Okamoto, A., Shikiji, W., Maeyama, K., Oike, H., Imaizumi, M. \& Yonezawa, N. Electrophilic aromatic aroylation polycondensation of acyl-acceptor and acyl-donor molecules composed binaphthylene or biphenylene aromatic ring assembly units. Kobunshi Ronbunshu 60, 147-153 (2009).

12 Miyaura, N. \& Suzuki, A. Palladium-catalyzed cross-coupling reactions of organoboron compounds. Chem. Rev. 95, 2457-2483 (1995).

13 Suzuki, A. \& Yamamoto, Y. Cross-coupling reactions of organoboranes: an easy method for C-C bonding. Chem. Lett. 40, 894-901 (2011).

14 Maeyama, K., Yamashita, K., Saito, H., Aikawa, S. \& Yoshida, Y. Synthesis of aromatic poly(ether ketone)s bearing optically active macrocycles through suzuki coupling polymerization. Polym. J. 44, 315-320 (2012)

15 Rehahn, M., Schluter, A.-D. \& Wegner, G. Pd-catalyzed polycondensation of aromatic monomers with functional groups. Makromol. Chem. Rapid Commun. 11, 535-539 (1990).

16 Higashibayashi, S. \& Sakurai, H. Synthesis of an enantiopure syn-benzocyclotrimer through regio-selective cyclotrimerization of a halonorbornene derivative under the palladium nanocluster conditions. Chem. Lett. 36, 18-19 (2007).

17 Higashibayashi, S. \& Sakurai, H. Asymmetric synthesis of a chiral buckybowl, trimethylsumanene. J. Am. Chem. Soc. 130, 8592-8593 (2008).

18 Reza, A. F. G. M., Higashibayashi, S. \& Sakurai, H. Preparation of $C_{3}$ symmetric homochiral syn-trisnorbornabenzenes through regio-selective cyclotrimerization of enantiopure iodonorbornenes. Chem. Asian. J. 4, 1329-1337 (2009).

19 Higashibayashi, S., Reza, A. F. G. M. \& Sakurai, H. Stereoselective cyclotrimerization of enantiopure iodonorbornenes catalyzed by pd nanoclusters for $c_{3}$ or $c_{3 v}$ symmetric syn-tris(norborneno)benzenes. J. Org. Chem. 75, 4626-4628 (2010).

20 Higashibayashi, S., Tsuruoka, R., Soujanya, Y., Purushotham, U., Sastry, G. N., Seki, S., Ishikawa, T., Toyota, S. \& Sakurai, H. Trimethylsumanene: enantioselective synthesis, substituent effect on bowl structure, inversion energy, and electron conductivity. Bull. Chem. Soc. Jpn. 85, 450-467 (2012).

21 Reetz, M. T., Breinbauer, R. \& Wanninger, K. Suzuki and heck reactions catalyzed by preformed palladium clusters and palladium/nickel bimetallic clusters. Tetrahedron Lett. 37, 4499-4502 (1996).

22 Lyubimov, S. E., Vasil'ev, A. A., Korlyukov, A. A., Ilyin, M. M., Pisarev, S. A., Matveev, V. V., Chalykh, A. E., Zlotin, S. G. \& Davankov, V. A. Palladium-containing hypercrosslinked polystyrene as an easy to prepare catalyst for Suzuki reaction in water and organic solvents. React. Funct. Polym. 69, 755-758 (2009).

23 Martins, D. L., Alvarez, H. M. \& Aguiar, L. C. S. Microwave-assisted Suzuki reaction catalyzed by $\mathrm{Pd}(0)-P V P$ nanoparticles. Tetrahedron Lett. 51, 6814-6817 (2010).

24 Ohtaka, A., Tamaki, Y., Igawa, Y., Egami, K., Shimomura, O. \& Nomura, R. Polyion complex stabilized palladium nanoparticles for suzuki and heck reaction in water. Tetrahedron 66, 5642-5647 (2010).

25 Hyotanishi, M., Isomura, Y., Yamamoto, H., Kawasaki, H. \& Obora, Y. Surfactant-free synthesis of palladium nanoclusters for their use in catalytic cross-coupling reactions. Chem. Commun. 47, 5750-5752 (2011).

26 Wang, W., Yang, Q., Zhou., R., Fu, H. -Y., Li, R. -X., Chen, H. \& Li, X. -J. Palladium nanoparticles generated from allylpalladium chloride in situ: a simple and highly efficient catalytic system for Mizoroki-Heck reactions. J. Organomet. Chem. 697, 1-5 (2012).

27 Maeyama, K., Tsukamoto, T., Suzuki, M., Higashibayashi, S. \& Sakurai, H. Synthesis of aromatic polyketones bearing 1,1'-binaphthyl-2,2'-dioxy units through suzuki-miyaura coupling polymerization. Chem. Lett. 40, 1445-1446 (2011). 